\title{
Comparison of ballistic and strength training on swimming turn and dry-land leg extensor characteristics in elite swimmers
}

\author{
Julian V Jones ${ }^{1,2}$, David B Pyne ${ }^{3,4}$, G Gregory Haff ${ }^{2}$ and \\ Robert U Newton ${ }^{2,5,6}$
}

\begin{abstract}
Swimmers undertake dry-land resistance training as part of their overall training regime in order to increase lower body force output, impulse and swim turn performance. We investigated whether short-term ballistic training or maximal strength training is more effective in enhancing leg extensor force characteristics during the swim turn. Twelve elite swimmers ( 10 males and 2 females 19.4 \pm 1.0 y) were assigned to either strength $(n=6)$ or ballistic leg extensor $(n=6)$ training based on their coaching group for a six-week period. All testing was conducted during the final training cycle towards the World Championships selection trials. Swimmers undertook dry-land testing of a squat jump on a portable force platform with bodyweight only and an additional $30 \mathrm{~kg}$ load for males and $20 \mathrm{~kg}$ load for females. On the same day, all swimmers performed a turn analysis using a fixed force platform within the pool wall. There were no substantial differences between the strength and ballistic groups after the six-week intervention. Only squat jump peak velocity (loaded) showed a moderately large standardized difference $(-0.7 \mathrm{I}, \pm 0.42 \mathrm{~m} / \mathrm{s})$ after six weeks in the strength-trained group. Relative peak power $(4.0 \pm 2.1 \mathrm{~W} / \mathrm{kg})$, squat jump peak force (loaded and unloaded) (195.0, $\pm 122.8 \mathrm{~N}$; $155.0, \pm 152.3 \mathrm{~N})$, and squat jump impulse (unloaded) $(2.9, \pm 2.1 \mathrm{~N}$ ) all showed small and clear improvements with ballistic training over the six-week intervention. Both strength and ballistic dry-land training can improve aspects of the push-off stage of the swim turn providing programming options for swimming and strength and conditioning coaches.
\end{abstract}

\section{Keywords}

Aquatic sport, force, power, resistance training, strength \& conditioning, vertical jump

\section{Introduction}

In high performance swimming, athletes dedicate many hours of training to attain small increments of improvement or marginal gains in overall performance. To stay in contention for an Olympic medal, a swimmer must improve his or her performance by $\sim 1 \%$ within a competition (heats to finals) and by $\sim 1 \%$ within the year leading up to the Olympics. ${ }^{1}$ To achieve these small increments of improvement, swimmers undertake both pool-based and dry-land training modalities to improve all aspects of their race performance. ${ }^{2}$ Swimming race performance can be divided into four key phases comprising the start, free swim, turns, and finish. ${ }^{3,4}$ The swimming turn has been further divided into a number of stages by multiple investigators, the most common being the five-stage model of: inward turn, rotation, push off, underwater, and outward turn. ${ }^{5}$
Reviewers: Lee Brown (California State University, Fullerton, USA)

Travis Triplett (Appachalian State University, USA)

'Strength \& Conditioning Discipline, Australian Institute of Sport, Bruce, Australia

${ }^{2}$ Centre for Exercise and Sport Science Research, Edith Cowan University, Joondalup, Western Australia

${ }^{3}$ Physiology Discipline, Australian Institute of Sport, Bruce, Australia

${ }^{4}$ Research Institute for Sport and Exercise, University of Canberra, Canberra, Australia

${ }^{5}$ School of Medical and Health Sciences, Edith Cowan University, Joondalup Western Australia

${ }^{6}$ The University of Hong Kong, Hong Kong, China

\section{Corresponding author:}

Julian V Jones, Strength \& Conditioning Discipline, Australian Institute of Sport, Leverrier Street, Bruce, ACT 2617, Australia.

Email: julian.jones@ausport.gov.au 
When examining the influence of the turn phase on swim performance, the majority of research has focused on the kinematics and kinetics of the different turn styles. ${ }^{6}$ Interestingly, almost all of these studies assert that increased application of force and impulse during the push-off stage of the turn by the swimmer can reduce total turn time. ${ }^{7}$ However, there is a paucity of studies examining the impact of longitudinal strength training interventions on the force-time characteristics of swim turn performance which can be examined collectively to clarify the contribution of the push-off phase to overall turning time and swimming performance.

Swimmers who exhibit faster overall turn times demonstrate significantly higher peak forces, ${ }^{8}$ reduced wall contact time ${ }^{9,10}$ and greater mean impulse ${ }^{10}$ than slower swimmers. The ability to generate higher propulsive forces during the push-off phase of a turn results in a higher final velocity off the wall. ${ }^{6}$ A reduction in overall turn time correlates highly $(\mathrm{r}=-0.90)$ with $50 \mathrm{~m}$ swim times. ${ }^{8}$ Thus, if a swimmer can increase their propulsive forces at a quicker rate, the impact of this higher force should promote faster competition performance.

During a normal training year, the swimmer has to contend with a large amount of concurrent training containing both pool and dry-land content which varies in accordance with the overall periodised training plan. ${ }^{11}$ Early in the year, pool training typically consists of a large volume of aerobic-based training followed by a greater focus on anaerobic training. Concurrently during this time, dry-land training focuses on developing strength before transitioning to ballistic training later in the season. This approach makes increasing the swimmer's force application capabilities difficult given a well-documented interference of concurrent strength and endurance training. ${ }^{12}$

One of the major differences between the concurrent training modalities applied to swimmers is that the volume of pool training is considerably higher than the dry-land component. Typically, swimmers undertake nine to ten $1.5-2 \mathrm{~h}$ pool sessions per week and only three $1-1.5 \mathrm{~h}$ dry-land sessions. Given the large bias towards pool training, gains in both strength and power might be compromised. ${ }^{13}$ In particular, Wilson et al. ${ }^{13}$ noted that athletes training to increase their rate of force development (RFD) are compromised more by concurrent training than just pure strength training due to the interference effect.

Dry-land training in swimmers has predominantly taken the approach of focusing on the development of both upper and lower body strength. A majority of dry-land studies have examined upper body power assessments in tethered swimming. ${ }^{14,15}$ The application of ballistic training for the lower limb in swimming performance has only been examined with plyometric activities ${ }^{16}$ or in swim starts. ${ }^{17}$ One method of training these qualities in swimmers is use of ballistic exercises as part of a comprehensive power development program. Maximal strength training involves heavy resistance, low repetitions, and consequently slow movement speed with emphasis on increasing the weight lifted. Ballistic training involves maximal effort against the resistance with the goal of maximal movement velocity, power output, and high RFD. ${ }^{18}$ Improvements in dry-land strength and power training should transfer to a more powerful and efficient swim turn. ${ }^{6}$ However, to the authors' knowledge, there are no studies that directly examined the effects of dryland strength and/or ballistic training on force-time and performance characteristics associated with swim turns in elite swimmers. Therefore, the purpose of this study was to determine whether ballistic training results in a greater improvement in the push-off stage of turn performance than a traditional maximal strength program.

\section{Methods}

\section{Subjects}

A total of 12 swimmers were assigned to a strengthtrained (ST) group or a ballistic-trained (BT) group based on existing coaching squads and then tested in the pool and on dry-land (Table 2). All swimmers were part of the Australian Institute of Sport (AIS) Swimming Squad with a minimum of two years of strength training experience. The strength group contained six swimmers consisting of four males and two females including a multi-Olympic medal winner and World Championship Team members. These swimmers undertook their normal dry-land strength training program over the course of the six-week training block. The BT contained six swimmers consisting of all males including an Olympic Medalist and World Championship Team members who completed a modified dry-land training program with a focus on ballistic leg extensor exercises. The swimmers of both groups performed three dry-land training sessions and 10 pool sessions per week. To assess the effect of the intervention, all swimmers were tested prior to and after the six-week training intervention on both a dry-land squat jump (SJ) protocol on a force plate and an in-water turn force plate protocol. The in-water protocol included measurement of key leg extensor force, impulse and kinematic characteristics over the $5 \mathrm{~m}$ approach to the wall and to the $5 \mathrm{~m}$ exit.

All swimmers were fully familiarized with the testing procedures, as they were part of their usual testing and training protocols. All tests were undertaken in the order of swim turn testing first followed by a minimum 
of one-hour rest and then the dry-land SJ test on the same day of the week.

All swimmers were tested as part of their normal training program in the final preparation period prior to taper (Figure 1) for World Championships selection trials (National Championships). This study was conducted in accordance with the Declaration of Helsinki and approved by the Ethics Committee of the AIS, Bruce, Australia and Edith Cowan University, Joondalup, Australia. All swimmers provided informed consent via their AIS Scholarship Agreement and signature of the relevant scholarship papers.

\section{Resistance training interventions}

The swimmers of the ST training group only performed strength exercises such as squats, dumbbell press, and

Table I. Characteristics of the swimmers in the strength and ballistic training groups at pre intervention.

\begin{tabular}{llcc}
\hline & & $\begin{array}{l}\text { Strength } \\
\text { trained } \\
\text { Mean } \pm \text { SD }\end{array}$ & $\begin{array}{l}\text { Ballistic } \\
\text { trained } \\
\text { Mean } \pm \text { SD }\end{array}$ \\
\hline Mass & $(\mathrm{kg})$ & $78.9 \pm 12.3$ & $77.1 \pm 10.2$ \\
Height & $(\mathrm{cm})$ & $179.1 \pm 8.6$ & $178.0 \pm 10.4$ \\
Age & $(\mathrm{y})$ & $19.4 \pm 1.1$ & $18.9 \pm 0.9$ \\
Training experience & $(\mathrm{y})$ & $8.3 \pm 3.1$ & $8.9 \pm 3.5$ \\
\hline
\end{tabular}

SD: standard deviation. chin ups without the intent of achieving a high velocity. In contrast, the swimmers of the BT training group performed ballistic exercises for the leg extensors with the intent to achieve a high velocity of movement (Table 2).

\section{Testing procedures}

Swim turn testing. Prior to conducting the swimming turn test, all swimmers completed a pool-based warm-up based on their prerace routine. This warm-up included some sprint, dive, and turn drills to ensure the swimmer was ready to perform all of their turns at maximal effort. Each swimmer then performed three maximal effort turns, with a 3-min rest period between each turn. The swimmer swam from $20 \mathrm{~m}$ out towards the wall at full speed, undertook their preferred stroke turn, touch or tumble, and swam at maximal effort back out to the $20 \mathrm{~m}$ mark. This distance allowed for capture of the full $15 \mathrm{~m}$ into the wall and back out to the $15 \mathrm{~m}$ mark as the full turn time. Kinematic and kinetic turn data were obtained using a proprietary force plate system ("Wetplate") instrumented and mounted within the pool wall. This system uses a wall-mounted Kistler force platform (Z20314, Winterthur, Switzerland) sampling at $500 \mathrm{~Hz}$. All kinetic characteristics of impulse, force, and relative peak power were filtered using a low-pass Butterworth digital filter with a cut-off frequency of $10 \mathrm{~Hz}$. Test-retest reliability for Wetplate was undertaken with a sub group of national squad swimmers yielding a percentage coefficient of

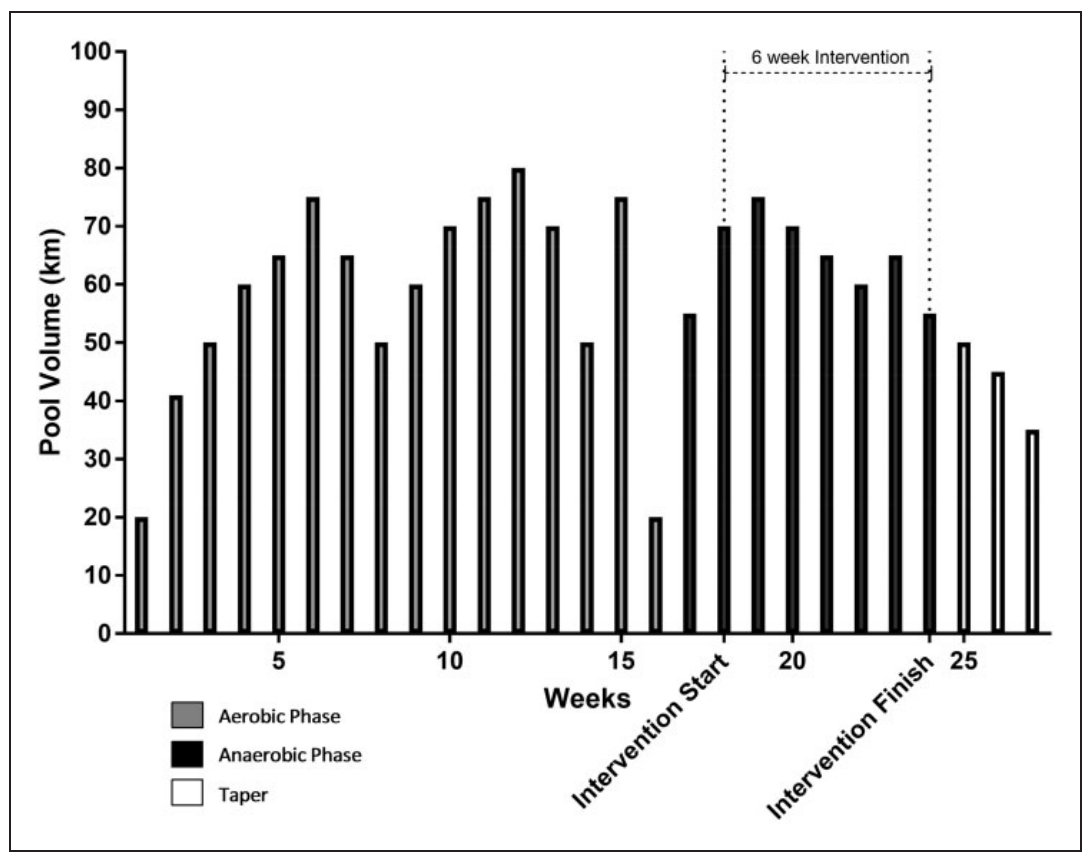

Figure I. Swimmers competition preparation plan with identified intervention period. 
Table 2. Characteristics of the dry-land training program by each group. ${ }^{a}$

\begin{tabular}{|c|c|c|c|c|c|}
\hline Exercises & Sets & Repetitions & Load (\% RM) & Rest (min) & $\begin{array}{l}\text { Sessions } \\
\text { per week }\end{array}$ \\
\hline \multicolumn{6}{|l|}{ Strength trained } \\
\hline $\begin{array}{l}\text { Bench press, leg press, bench pull, shoulder press, } \\
\text { chin ups, and squats }\end{array}$ & $4-5$ & $5-8$ & $85-90$ & $3-4$ & 3 \\
\hline \multicolumn{6}{|l|}{ Ballistic trained } \\
\hline $\begin{array}{l}\text { Power cleans, push press, jump squats, box jumps, } \\
\text { and medicine ball throws }\end{array}$ & $4-5$ & $3-5$ & $80-100$ & $2-3$ & 3 \\
\hline
\end{tabular}

RM: repetition maximum.

${ }^{a}$ All dry-land training sessions were undertaken I.5 h post pool training in the morning. Training sessions were undertaken on Monday, Wednesday, and Friday of each week.

variation $(\% \mathrm{CV})$ of $2.4-4.7 \%$ across the four characteristics measured.

Dry-land testing. The SJ was chosen as there is no preparatory countermovement and thus the stretch shortening cycle contribution is negated. A number of studies have shown that the "push-off" phase, which is concentric extension only, ${ }^{4,19,20}$ makes up the largest percentage of wall contact time.

SJ protocol. Swimmers undertook a standardized warmup consisting of $5 \mathrm{~min}$ of light aerobic activity performed on a stationary bicycle maintaining a cadence of $80 \mathrm{r} / \mathrm{min}$, a predetermined series of dynamic joint range of motion, and static stretches for the quadriceps and hamstring muscle groups. This work was followed by two sets of five repetitions of box jumps, and two sets of five repetitions of the power clean performed with an unloaded barbell $(20 \mathrm{~kg})$ to prepare the athlete for the subsequent jump testing.

After completion of the standardized warm-up, all participants performed three unloaded and three loaded ( $30 \mathrm{~kg}$ for males and $20 \mathrm{~kg}$ for females) maximal effort SJs, with a 2-min rest period between each jump while standing on a uniaxial force plate (400 Series Performance Force Plate, Fitness Technology, Australia) interfaced with commercially available computer software (Ballistic Measurement System, Innervations, Australia). Squat or concentric-only jumps were used as they more closely mimic the predominantly concentric muscle actions evident in the swim turn. All force data were collected at $200 \mathrm{~Hz}$ and filtered using a low-pass Butterworth digital filter with a cut-off frequency of $50 \mathrm{~Hz}$. All force-time curves collected during the jump trials (loaded and unloaded) were then analyzed for peak force, peak velocity, peak power, impulse, and peak power per kg of body mass. Each swimmer was instructed to lower their body to the correct start position of the SJ and hold for a count of three $\mathrm{s}$ before performing the jump. Each swimmer's jump force trace was checked to ensure there was no countermovement prior to the concentric-only action. A test-retest reliability for barbell-loaded SJ characteristics in our laboratory has been established as a $2.2 \% \mathrm{CV}^{21}$

\section{Statistical analyses}

Descriptive data are reported as mean and standard deviation (SD). Inferences on differences in the mean change in strength/power characteristics between the ST and BT groups from week one to week six were made using a Student's $t$ test for dependent samples. Precision of estimation was indicated with $90 \%$ confidence limits. Effect sizes were reported in standardized (Cohen's $d$ ) units as the change in the mean with $90 \%$ confidence limits. Criteria to assess the magnitude of observed changes were: $0.0-0.2$ trivial; $0.20-0.60$ small; $0.60-1.20$ moderate; and >1.20 large. An effect was deemed unclear if its confidence interval spanned both substantial positive and substantial negative values $\left( \pm 0.20 \mathrm{x}\right.$ between subject SD) ${ }^{22}$ A power analysis was undertaken to ascertain if the intervention had enough subjects to confidently determine a significant difference. $^{22}$ A sample size of six was deemed sufficient to identify a reference change of $4.4 \%$ in SJ peak velocity, assuming a typical error of $1.7 \%$.

\section{Results}

The intervention period of six weeks resulted in the BT showing a substantial within-group improvement in the in-pool test characteristic of peak power per $\mathrm{kg}$ (Table 3). The BT also showed substantial withingroup improvements in the dry-land test characteristics of peak force loaded and unloaded. The remaining 12 characteristics measured from both pool and dry-land tests showed no substantial within-group changes over the six weeks. There was, however, a large difference at baseline between the BT and ST in relative squat strength; BT had 50\% higher (standardized difference $1.79 \pm 0.98$; mean $\pm 90 \%$ confidence limits) relative 
Table 3. Comparison of six weeks of ballistic training compared with strength training in swimming turn and dry-land leg extensor characteristics. $^{\text {a }}$

\begin{tabular}{|c|c|c|c|c|c|c|c|}
\hline & \multicolumn{3}{|l|}{ Strength trained } & \multicolumn{3}{|l|}{ Ballistic trained } & \multirow{2}{*}{$\begin{array}{l}\begin{array}{l}\text { Standardized } \\
\text { difference }\end{array} \\
\text { Between } \\
\text { groups ES, } \\
\pm 90 \% \mathrm{CL}\end{array}$} \\
\hline & $\begin{array}{l}\text { Week I } \\
\text { Mean } \pm \text { SD }\end{array}$ & $\begin{array}{l}\text { Week } 6 \\
\text { Mean } \pm \text { SD }\end{array}$ & $\begin{array}{l}\text { Change Mean, } \\
\pm 90 \% \mathrm{CL}\end{array}$ & $\begin{array}{l}\text { Week I } \\
\text { Mean } \pm \text { SD }\end{array}$ & $\begin{array}{l}\text { Week } 6 \\
\text { Mean } \pm \text { SD }\end{array}$ & $\begin{array}{l}\text { Change Mean, } \\
\pm 90 \% \mathrm{CL}\end{array}$ & \\
\hline \multicolumn{8}{|c|}{ Swim turn characteristics-Wetplate } \\
\hline Impulse (n) & $3.3 \pm 1.0$ & $4.0 \pm 0.3$ & $0.7 \pm 1.1$ & $4 \pm 0.3$ & $4.2 \pm 0.3$ & $0.04, \pm 0.4$ & $0.12, \pm 0.4$ \\
\hline Turn time $(\mathrm{s})$ & $8.1 \pm 0.8$ & $8.0 \pm 0.8$ & $-0.1 \pm 0.4$ & $8.5 \pm 1.0$ & $8.4 \pm 0.9$ & $0.0, \pm 0.4$ & $0.15, \pm 0.5$ \\
\hline Time to $5 \mathrm{~m} \mathrm{(s)}$ & $2.2 \pm 0.6$ & $2.3 \pm 0.6$ & $0.1 \pm 0.4$ & $2.7 \pm 0.7$ & $2.5 \pm 0.5$ & $-0.19, \pm 0.4$ & $-0.20, \pm 0.6$ \\
\hline $\begin{array}{l}\text { Peak power per } \\
\mathrm{kg}(\mathrm{W} / \mathrm{kg})\end{array}$ & $49.7 \pm 19.2$ & $52.3 \pm 18.3$ & $2.6 \pm 9.9$ & $62.1 \pm 6.7$ & $66.1 \pm 5.2$ & $4.0, \pm 2.1^{b}$ & $0.15, \pm 0.49$ \\
\hline \multicolumn{8}{|c|}{ Dry-land characteristics—squat jump } \\
\hline $\begin{array}{l}\text { Relative squat } \\
\text { strength }(\mathrm{kg} / \mathrm{kg})\end{array}$ & $0.82 \pm 0.20$ & $0.8 \pm 0.17$ & $0.0, \pm 0.06$ & $1.2 \pm 0.2$ & $1.2 \pm 0.2$ & $0.0, \pm 0.0$ & $0.13, \pm 0.27^{c}$ \\
\hline $\begin{array}{l}\text { Peak power per } \\
\text { kg L (W/kg) }\end{array}$ & $44.9 \pm 8.4$ & $44.7 \pm 8.6$ & $-0.2, \pm 6.1$ & $67.1 \pm 9.6$ & $61.1 \pm 7.8$ & $-6.0, \pm 6.7$ & $0.09, \pm 0.67$ \\
\hline $\begin{array}{c}\text { Peak power per } \\
\text { kg UL (W/kg) }\end{array}$ & $47.1 \pm 19.4$ & $48.5 \pm 10.2$ & $1.4, \pm 10.5$ & $62.7 \pm 8.6$ & $68.2 \pm 8.1$ & $5.5, \pm 16.7$ & $0.21, \pm 0.76$ \\
\hline Impulse $(\mathrm{N}) \mathrm{L}$ & $107.9 \pm 15.3$ & $110.1 \pm 12.3$ & $2.1, \pm 10.2$ & $114.3 \pm 4.2$ & $117.2 \pm 7.3$ & $3.0, \pm 5.0$ & $0.05, \pm 0.67$ \\
\hline Impulse (N) UL & $78.4 \pm 12.8$ & $83.4 \pm 9.0$ & $5.0, \pm 10.2$ & $85.1 \pm 5.2$ & $88.0 \pm 6.1$ & $2.9, \pm 2.1^{\mathrm{b}}$ & $-0.08, \pm 0.68$ \\
\hline Peak force $(N) L$ & $1854.3 \pm 316.0$ & $1961.9 \pm 312.3$ & $107.7, \pm 163.8$ & $2151.5 \pm 154.7$ & $2346.5 \pm 221.7$ & $195.0, \pm 122.8^{b}$ & $0.19, \pm 0.55$ \\
\hline Peak force $(N)$ UL & $1635.5 \pm 313.6$ & $1770.7 \pm 332.7$ & $135.3, \pm 211.6$ & $1939.6 \pm 175.7$ & $2094.6 \pm 212.2$ & $155.0, \pm 152.3^{b}$ & $0.16, \pm 0.7$ \\
\hline Peak power $(\mathrm{W}) \mathrm{L}$ & $3620.2 \pm 782.4$ & $3652.0 \pm 984.3$ & $31.8, \pm 510.7$ & $5511.6 \pm 656.5$ & $5059.0 \pm 730.3$ & $-452.6, \pm 515.0$ & $-0.23, \pm 0.73$ \\
\hline Peak power $(\mathrm{W}) \mathrm{UL}$ & $3818.3 \pm 1617.3$ & $3930.3 \pm 875.2$ & $111.5, \pm 783.0$ & $5177.7 \pm 856.8$ & $5630.1 \pm 662.4$ & $452.5, \pm 1395.3$ & $0.65, \pm 0.21^{d}$ \\
\hline Peak velocity $(\mathrm{M} / \mathrm{S}) \mathrm{L}$ & $2.1 \pm 0.2$ & $2.1 \pm 0.3$ & $-0.01, \pm 0.3$ & $2.9 \pm 0.3$ & $2.5 \pm 0.2$ & $-0.4, \pm 0.2$ & $-0.32, \pm|.5|$ \\
\hline Peak velocity $(\mathrm{M} / \mathrm{S}) \mathrm{UL}$ & $2.5 \pm 0.7$ & $2.8 \pm 0.6$ & $0.3, \pm 0.6$ & $3.2 \pm 0.6$ & $3.2 \pm 0.3$ & $-0.0, \pm 0.9$ & $0.36, \pm 0.55^{\mathrm{e}}$ \\
\hline Mass $(\mathrm{kg})$ & $80.4 \pm 7.3$ & $80.9 \pm 8.0$ & $0.5, \pm 1.8$ & $82.4 \pm 4.2$ & $82.7 \pm 4.5$ & $0.3, \pm 1.1$ & $-0.04, \pm 0.26$ \\
\hline
\end{tabular}

CL: Confidence Limit; ES: effect statistic; L: loaded; UL: unloaded; SD: standard deviation.

${ }^{a}$ There was a substantial improvement in peak power per $\mathrm{kg}$ in both groups over the six weeks. Standardized difference indicates the mean (standardized) difference between the groups in the change in mean value from week one to week six.

${ }^{b}$ Denotes substantial within-group improvement.

'Denotes trivial effect.

${ }^{\mathrm{d} D e n o t e s}$ moderate effect.

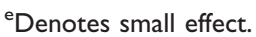

squat strength. No substantial changes within or between groups occurred in relative squat strength over the six-week intervention.

There was only one characteristic with a substantial difference in the mean changes between ST and BT groups in selected strength/power characteristics in either the pool or dry-land testing the SJ peak power UL, a moderate effect. There was a substantially greater increase in SJ peak velocity UL within the ST group. Within the three repeat jump protocol, the mean Intraclass Correlation Coefficient (ICC) was 0.96 for SJ peak velocity UL and 1.00 for SJ peak power UL and 0.99 for SJ peak velocity L and 1.00 for SJ peak power L. The BT showed small improvements in one pool characteristic peak power per $\mathrm{kg}$ and three dry-land characteristics: impulse L, peak force L and UL. Only the SJ peak velocity was improved in response to the six-week training period in the ST group. All other characteristics measured in both the BT and ST had unclear effects (Table 3).

\section{Discussion}

The primary finding of the study was that there were no demonstrable differences in performance of the pushoff phase of a swim turn after completion of six weeks of training between the BT or ST groups. Each group exhibited one or two substantial within-group changes in either in-pool or dry-land characteristics. The failure to elicit larger improvements and in a wider range of strength/power characteristics over the six weeks indicates the challenges for physical development late in the competitive swimming season. Possible explanations for the variable level of improvements include the short length of time of the intervention, the effect of concurrent training, the associated optimal recovery 
time periods and a relatively low level of strength at the start of the training intervention, in particular, for the ST group.

The BT group had small improvements in response to the six-week intervention period in only one pool characteristic (relative power output) and three dry-land characteristics (impulse loaded, peak force loaded, and unloaded). Given that at the elite levels of swimming, only $\sim 1 \%$ change in performance is expected over a competition year ${ }^{1}$ relatively small improvements would be expected with a short intervention period. A number of swimming studies have examined concurrent training and reported that the time taken to gain a meaningful shift can be substantially longer in the presence of competing physiological demands than training in one modality alone. ${ }^{23-25}$

Izquierdo-Gabarren et al. $^{26}$ reported that eight weeks of concurrent strength and endurance training with rowers using a moderate number of repetitions (not to failure) yielded greater improvements in strength and muscle power than the group who undertook training to failure. ${ }^{26}$ In the present study, the subjects also did not train to failure in their dry-land strength or ballistic training and undertook similar volumes of concurrent training. In a study by Baar, ${ }^{27}$ the authors reported performing strength training immediately after a low-intensity volume session results in a greater stimulus for volume adaptation than the lowintensity volume session alone. Taken together, these data provide greater support for the rationale that optimizing recovery to limit cumulative fatigue is an important consideration when undertaking concurrent training not to train to failure/depletion in either the strength/ballistic dry-land or pool training sessions. These studies were undertaken with upper body interventions only, but followed a similar time course (six and eight weeks, respectively) to this study. ${ }^{28}$

The initial strength level of a swimmer could influence the rate of improvement in strength/power characteristics. West et al. ${ }^{17}$ reported that elite swimmers should have a strength capability of 1.7 times bodyweight in one repetition maximum (RM) relative squat strength to improve swim performance. No swimmers in this study had a high level of strength compared with other well-trained athletes given the modest relative squat strength. No substantial effect on the dryland force characteristics within or between the groups occurred during this intervention. Resistance training interventions that focus on strength development would potentially result in a larger adaptation. Cormie et al. ${ }^{29}$ also reported that weaker strengthtrained college athletes with a squat of less than 1.3 times bodyweight in one RM relative squat strength obtained greater gains in both strength and power when targeting strength development. Given that the BT had a higher relative squat strength to the ST at baseline, the outcome of this intervention is not inconsistent with the outcomes of the Cormie et al. study. It appears the swimmers in this study needed a combination of strength and ballistic training to enhance or maintain their strength foundation.

Scheduling of the different types of training to optimize the recovery time, and minimize the interference from competing modalities, is important when prescribing the daily and weekly training regime. ${ }^{30}$ The programming of the different types of training during this intervention was scheduled only $1.5 \mathrm{~h}$ apart and the limited time might have compromised the swimmers' ability to elicit larger capacity changes with insufficient recovery. Recently, Murach and Bagley ${ }^{30}$ suggest that a minimum of six $h$ of recovery should be allotted between concurrent training methods to minimize the interference effect. Further reductions in the impact of the interference effect are expected if $24 \mathrm{~h}$ can be allotted between training factors. These points indicate that the coach should prioritize pool, dry-land, and recovery training depending on the primary objective(s) of the training phase,

It is also possible concurrent training prevented an increase in muscle mass (hypertrophy). ${ }^{27} \mathrm{~A}$ minimum of six $\mathrm{h}$ is needed between factors to minimize the negative impact of concurrent training on muscle hypertrophy. ${ }^{30}$ Without hypertrophy, a key component to increasing overall strength levels, a swimmer's ability to make both strength and power gains would be limited. ${ }^{31}$ While it is not desirable for swimmers to gain size, given its adverse effect on drag, increasing the underlying strength enables a greater power output and ultimately performance. This study did not undertake body composition testing over the six-week intervention period and along with no significant change in body mass, the influence of size warrants further investigation. We acknowledge the outcomes of this investigation represent a composite analysis of male and female subjects - further gender specific work is required to more closely define the effects of strength and power interventions within and between the genders in swimming.

Power could be most affected by concurrent training. Wilson et al. ${ }^{13}$ speculated that decrements in power result from either impairments of contraction velocity or RFD. This may account for the small changes observed in the BT group over the intervention period. It also might explain why other studies ${ }^{32}$ did not find any positive effects from plyometric training on performance in swimmers. The ability of swimmers to respond to ballistic training can be limited given the volume of swim training that they carry out for long periods of time. ${ }^{33}$ The prolonged periods of volume of greater than $5 \mathrm{~km}$ per day at certain times of the preparation cycle can inhibit a swimmer's capacity to recruit 
large motor units and fast-twitch muscle fibers. ${ }^{12,34}$ Given the current intervention was during the final full training preparation phase, the expectation was that the mix of training activities would promote shifts in recruitment of larger motor units. Unfortunately, the final phase of training could not be extended to allow for greater time exposure to the intervention given the upcoming competition.

\section{Conclusion}

There was little difference between strength and ballistic leg extensor training on swim turn performance in elite swimmers preparing for major competition. Future studies should consider the amount of concurrent training undertaken if the priority is to enhance strength and power qualities. Consideration of the optimal placement of the strength training priority training cycle needs to be undertaken in the context of the overall yearly training plan as there are limitations to achieving strength gains when the swimmers are in-season. Swimmers need a base level of strength prior to implementing ballistic exercise for best effect. Longer interventions with single modality trained athletes might promote larger improvements in strength/power and swim turn performance.

\section{Practical applications}

As a strength and conditioning coach, it has to be accepted that a swimmer will undertake concurrent training the majority of training year. To enable the swimmer to make significant gains in their ballistic leg extensor characteristics, a substantial proportion of time (perhaps longer than six weeks later in the season) is needed to enhance these characteristics without the interference of a high pool training volume. It appears that $>1.5 \mathrm{~h}$ of recovery should be allocated between gym and pool training and up to six $\mathrm{h}$ would be ideal. The swim coach must collaborate with the strength and conditioning coach to optimize a swimmer's adaptation to both the pool and dry-land training programs.

\section{Acknowledgements}

The authors acknowledge the cooperation of members of the AIS Swimming Team and assistance of swim coaches Ms. Tracey Menzies and Mr. John Fowlie. The authors also wish to thank Dr. Dave Pease (Aquatic Testing and Training Unit, Australian Institute of Sport) for his and his staff's valuable assistance in the collection of turn data.

\section{Declaration of Conflicting Interests}

The author(s) declared no potential conflicts of interest with respect to the research, authorship, and/or publication of this article.

\section{Funding}

The author(s) received no financial support for the research, authorship, and/or publication of this article.

\section{References}

1. Pyne DB, Trewin CB and Hopkins WG. Progression and variability of competitive performance of olympic swimmers. J Sports Sci 2004; 22: 613-620.

2. Aspenes S, Kjendlie PL, Hoff J, et al. Combined strength and endurance training in competitive swimmers. $J$ Sports Sci Med 2009; 8: 357-365.

3. Mason BR and Fowlie JK. Competition analysis for high performance swimming. In: Australian swimming coaches association, Australasian-oceania swimming professionals convention \& trade exhibition: From infants to internationals: 1997 proceedings, [Australia], [asca], 1997, p.65-70.

4. Chakravorti N, Slawson SE, Cossor J, et al. Swimming turn technique optimisation by real-time measurement of foot pressure and position. Procedia Eng 2012; 34: 586-591.

5. Mason B. Biomechanical race analysis. Swim Aust 1999; 15: 29-39.

6. Lyttle AD and Mason B. A kinematic and kinetic analysis of the freestyle and butterfly turns. J Swim Res 1997; 12: 7-11.

7. Wakayoshi K, Nomura T, Takahashi G, et al. Analysis of swimming races in the 1989 Pan Pacific Swimming Championships and 1988 Japanese Olympic Trials. In: Maclaren D (ed.) Biomechanics and medicine in swimming: swimming science VI, 1st ed. London: E \& FN Spon, 1992, pp.135-141.

8. Blanksby BA, Hodgkinson JN and Marshall RN. Forcetime characteristics of freestyle tumble turns by elite swimmers. SA J Res Sport 1996; 19: 1-15.

9. Chow JWC, Hay JG, Wilson BD, et al. Turning techniques of elite swimmers. J Sports Sci 1984; 2: 241-255.

10. Takahashi G, Yoshida A, Tsubakimoto S, et al. Propulsive force generated by swimmers during a turning motion. In: Hollander AP (ed.) Biomechanics and medicine in swimming: Proceedings of the Fourth International Symposium of Biomechanics in Swimming and the Fifth International Congress on Swimming Medicine held in Amsterdam, The Netherlands, June 21-25, 1982. Champaign, IL: Human Kinetics Publishers, 1983, pp.192-198.

11. Haycraft $\mathbf{J}$ and Robertson $\mathrm{S}$. The effects of concurrent aerobic training and maximal strength, power and swimspecific dry-land training methods on swim performance: a review. J Aust Str Con 2015; 23: 91-99.

12. Garrido N, Marinho DA, Reis VM, et al. Does combined dry land strength and aerobic training inhibit performance of young competitive swimmers? J Sports Sci Med 2010; 9: 300-310.

13. Wilson JM, Marin PJ, Rhea MR, et al. Concurrent training: a meta-analysis examining interference of aerobic and resistance exercises. J Str Con Res 2012; 26: 2293-2307. 
14. Loturco I, Barbosa AC, Nocentini RK, et al. A correlational analysis of tethered swimmimg, swim sprint performance and dry-land power assessments. Int $J$ Sports Med 2015; 37: 211-218.

15. Gergley TJ, McArdle WD, DeJesus P, et al. Specificity of arm training on aerobic power during swimming and running. Med Sci Sports Exerc 1984; 16: 349-354.

16. Cossor JM, Blanksby BA and Elliott BC. The influence of plyometric training on the freestyle tumble turn. $J \mathrm{Sci}$ Med Sport 1999; 2: 103-116.

17. West DJ, Owen NJ, Cunningham DJ, et al. Strength and power predictors of swimming starts in international sprint swimmers. J Str Con Res 2011; 25: 950-955.

18. Cormie P, McCaulley GO and McBride JM. Power versus strength-power jump squat training: influence on the load-power relationship. Med Sci Sports Exerc 2007; 39: 996-1003.

19. Lyttle A. Investingating kinetics in the freestyle flip turn push off. J Appl Biomech 1999; 15: 242-252.

20. Zatsiorsky VM and Kraemer WJ. Science and practice of strength training. Champaign, IL: Human Kinetics, 2006.

21. Sheppard JM and Doyle TLA. Increasing compliance to instructions in the squat jump. J Str Con Res 2008; 22: 648-651.

22. Hopkins WMS, Batterham A and Hanin J. Progressive statistics for studies in sports medicine and exercise science. Med Sci Sports Exerc 2009; 41: 3-12.

23. Morouço P, Neiva H, González-Badillo JJ, et al. Associations between dry land strength and power measurements with swimming performance in elite athletes: a pilot study. J Hum Kinetics 2011; 29A: 105-112.

24. Tanaka H, Costill DL, Thomas R, et al. Dry-land resistance training for competitive swimming. Med Sci Sports Exerc 1993; 25: 952-959.
25. Counsilman D. The residual effects of training. Swim Aust 2014; 30: 25-34.

26. Izquierdo-Gabarren $M$, De Txabarri Expósito RG, García-Pállarés $\mathrm{J}$, et al. Concurrent endurance and strength training not to failure optimizes performance gains. Med Sci Sports Exerc 2010; 42: 1191-1199.

27. Baar K. Using molecular biology to maximize concurrent training. Sports Med (Auckland, NZ) 2014; 44: S117-S125.

28. Bahadoran MR. The effect of the sequence of concurrent strength and endurance training on the flip turn. World Appl Sci J 2012; 17: 1120-1124.

29. Cormie P, McGuigan MR and Newton RU. Influence of strength on magnitude and mechanisms of adaptation to power training. Med Sci Sports Exerc 2010; 42: 1566-1581.

30. Murach KA and Bagley JR. Skeletal muscle hypertrophy with concurrent exercise training: contrary evidence for an interference effect. Sports Med (Auckland, NZ) 2016; 46: 1029-1039.

31. Aagaard P and Andersen JL. Effects of strength training on endurance capacity in top level endurance athletes. Scand J Med Sci Sport 2010; 20: 39-47.

32. Potdevin FJ, Alberty ME, Chevutschi A, et al. Effects of a 6-week plyometric training program on performances in pubescent swimmers. J Str Con Res 2011; 25: 80-86.

33. Mujika I, Padilla S and Pyne D. Swimming performance changes during the final 3 weeks of training leading to the Sydney 2000 Olympic Games. Int J Sports Med 2002; 23: 582-587.

34. Garrido N, Marinho DA, Barbosa TM, et al. Relationships between dry land strength, power variables and short sprint performance in young competitive swimmers. J Hum Sport Exerc 2010; 5: 240-249. 